\title{
Infrared and Raman Microscopy: Pushing the Limits of Spatial Resolution
}

Thomas J Tague Jr.

Bruker Optics, Inc., 19 Fortune Drive, Billerica, MA 01821

Historically, infrared microanalysis has been a valuable tool assisting in the chemical characterization of samples. Most analytical laboratories have employed infrared microscopy for many years. More recently, infrared microscopes have been designed to incorporate optical microscopy capabilities to additionally allow for the physical characterization of the given sample. Raman microscopes have also been available for several years and found utility in performing spectroscopic analysis down to 1 micron.

In comparing the spatial resolution of infrared and Raman microscopes, the following differences are readily evident. For infrared microanalysis, the spatial resolution is diffraction limited by the wavelength of light and the numerical aperture of conventionally used reflecting objectives. Diffracted light is projected one wavelength of light away from the origin, as shown in Figure 1. Previously, infrared microscopes that used single point detectors with remote masks (apertures) suffered from light loss and diffraction to such an extent that the observation of diffraction rings was unobserved.

Figure 2 shows the expected diffraction rings collected from a thin bone sample and 5 micron ink spot, respectively. The ink spots have been deposited onto a $\mathrm{KBr}$ salt window using a piezo ink delivery device to allow for the characterization of isolated particles. A focal-plane array detector was utilized to provide full field detection, thereby alleviating the use of the light attenuating remote field mask. At wavelengths shorter than the desired spatial resolution, no diffraction rings are observed and at wavelengths longer than the spatial resolution diffraction rings are observed. The $\mathrm{CH}$ stretching region was used for the shorter wavelength profile and the $1420 \mathrm{~cm}-1$ band was used to profile the longer wavelengths. The bone sample was prepared by cryogenic microtome after mounting in a paraffin medium. The total collection time for the infrared images was 1 minute at $4 \mathrm{~cm}^{-1}$ spectral resolution using a standard SiC globar source.

For dispersive Raman microscopy, the numerical aperture of refraction-based lenses is larger and the excitation wavelength is short resulting in at least 5 times better spatial resolution. The analysis area for Raman microanalysis is typically defined by the laser spot size. Patterned carbon nanotubes arrays of have been analyzed to determine how small an area can be imaged utilizing Raman microscopy.

Infrared microscopes are unable to perform confocal depth analysis, where modern Raman microscopes incorporate a confocal design to allow depth profiling of materials. A novel and patented confocal design has been employed that utilizes an array of pinholes and slits at the spectrograph entrance, thereby providing a depth resolution of better than 1 micron. Examples of multilayer polymer laminates will be shown 
demonstrating the benefit of high resolution depth profiling, alleviating the necessity for cross-sectional analysis.

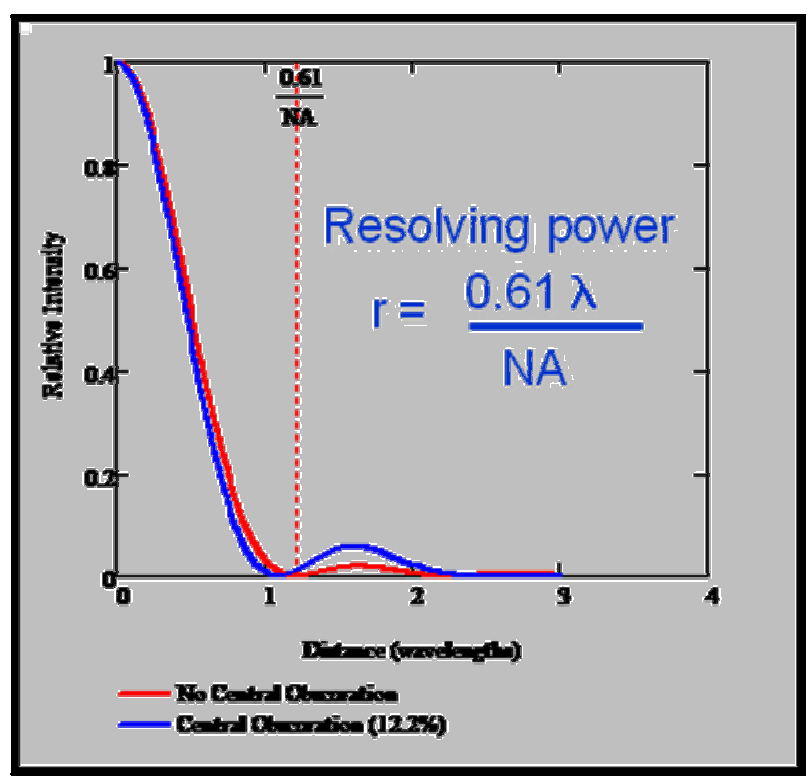

Figure 1 - The effects of diffraction as a function of distance from the origin.
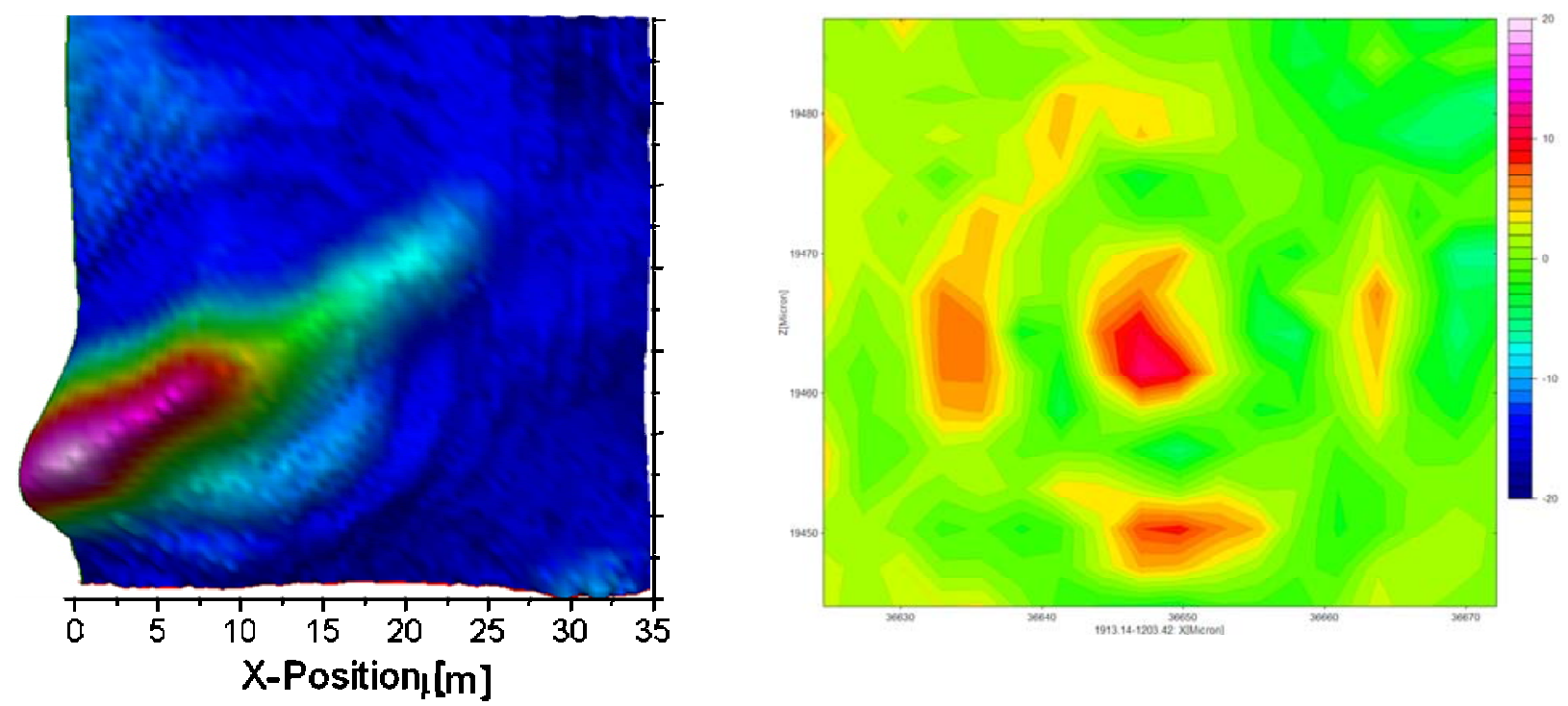

Figure 2 - The left image is of a bone sample integrated at $1420 \mathrm{~cm}^{-1}$ and the right image is the integrated image of a small ink spot. 